OPEN ACCESS

Edited by: Chandan Kumar

University of Michigan, United States

Reviewed by:

Marianna Aprile,

Italian National Research Council

(CNR), Italy

Yuji Kageyama

Kobe University, Japan

${ }^{*}$ Correspondence:

Doreen Ware

ware@cshl.edu

Specialty section: This article was submitted to

RNA,

a section of the journal

Frontiers in Genetics

Received: 30 November 2018

Accepted: 09 April 2019

Published: 26 April 2019

Citation:

Wang B, Kumar V, Olson A and

Ware D (2019) Reviving

the Transcriptome Studies: An Insight

Into the Emergence

of Single-Molecule Transcriptome

Sequencing. Front. Genet. 10:384.

doi: 10.3389/fgene.2019.00384

\section{Reviving the Transcriptome Studies: An Insight Into the Emergence of Single-Molecule Transcriptome Sequencing}

\author{
Bo Wang ${ }^{1}$, Vivek Kumar' ${ }^{1}$, Andrew Olson ${ }^{1}$ and Doreen Ware ${ }^{1,2 *}$ \\ ${ }^{1}$ Cold Spring Harbor Laboratory, Cold Spring Harbor, NY, United States, ${ }^{2}$ USDA-ARS Robert W. Holley Center for \\ Agriculture and Health, Ithaca, NY, United States
}

Advances in transcriptomics have provided an exceptional opportunity to study functional implications of the genetic variability. Technologies such as RNA-Seq have emerged as state-of-the-art techniques for transcriptome analysis that take advantage of high-throughput next-generation sequencing. However, similar to their predecessors, these approaches continue to impose major challenges on full-length transcript structure identification, primarily due to inherent limitations of read length. With the development of single-molecule sequencing (SMS) from PacBio, a growing number of studies on the transcriptome of different organisms have been reported. SMS has emerged as advantageous for comprehensive genome annotation including identification of novel genes/isoforms, long non-coding RNAs and fusion transcripts. This approach can be used across a broad spectrum of species to better interpret the coding information of the genome, and facilitate the biological function study. We provide an overview of SMS platform and its diverse applications in various biological studies, and our perspective on the challenges associated with the transcriptome studies. Keywords: transcriptomics, RNA-Seq, Iso-Seq, single-molecule transcriptome sequencing, alternative
splicing, isoforms

\section{INTRODUCTION}

The last few decades have witnessed an explosive growth in the genomic sequencing technologies (Shendure et al., 2017). As a result of increased throughput, higher accuracies and lower costs, there has been an exponential growth in genomic sequence databases over the last two decades (Lathe et al., 2008; Heather and Chain, 2016; Levy and Myers, 2016; Ardui et al., 2018; KarschMizrachi et al., 2018). However, a major challenge in the molecular biology continues to be the complex mapping of the same genome to diverse phenotypes in different tissue types, development stages and environmental conditions. A better understanding of the transcripts and expression of gene regulation is not only non-trivial but lies at the heart of this challenge. Transcriptomics offers important insights on gene structure, expression, and regulation and has been widely studied in many organisms (Jain, 2012; Casamassimi et al., 2017; Lowe et al., 2017). The transcriptomics studies have advanced considerably because of the explosive growth in the underlying sequencing technology (Abdel-Ghany et al., 2016; Wang et al., 2016). 
Our objective here is to outline the current standards and resources for the platform and the bioinformatics approaches underlying the transcript profiling. We also aim to provide an overview of the single-molecule transcriptome sequencing workflow, particularly PacBio Iso-Seq, and briefly discuss various tools at different stages of the workflow. While we cover the broader technology landscape in this paper, we do not aim to provide an exhaustive compilation of resources or software tools or a highlight of the select tools. We finally conclude with a brief discussion of the opportunities as well as challenges associated with long read transcript profiling as compared to traditional short read techniques such as RNA-Seq.

\section{EVOLUTION OF SEQUENCING TECHNOLOGIES}

First generation sequencing is primarily represented by the DNA sequencing approach pioneered by Sanger and Coulson, 1975 and is based on the selective incorporation of chainterminating dideoxynucleotides by DNA polymerase during in vitro replication of DNA (Sanger and Coulson, 1975, Sanger et al., 1977). Another DNA sequencing approach was developed a year later by Maxam and Gilbert (1977) which was based on partial chemical modification of DNA specific to nucleotide bases and a subsequent cleavage of the DNA backbone at sites adjacent to the modified nucleotides. Unlike Sanger approach which required cloning to generate single strand DNA, MaxamGilbert sequencing was advantageous since it could directly use the purified DNA (Saccone and Pesole, 2003). However, Sanger's chain termination method proved to be relatively easier to scale with the improvement of the chain-termination method and was widely used for next three decades including for the first draft of the Human Genome project. While it could sequence DNA fragments as long as $1 \mathrm{~kb}$ with a high raw read accuracy, it was limited by the low throughput and high cost (Schloss, 2008).

Second generation of sequencing (SGS) alternatively referred as next generation sequencing (NGS) technology, originated in mid 2000s to support massively parallel sequencing of hundreds of thousands of short DNA strands that are anchored and read through multiple "wash and scan" cycles (Moorthie et al., 2011; Goodwin et al., 2016). For example, Illumina HiSeq platforms can generate upward of 5 billion reads and $1500 \mathrm{~Gb}$ per run. Also, this approach is able to generate high read accuracy with much lower cost. However, the reads are generally limited in length to couple of $100 \mathrm{~s}$ of bases because of incremental errors introduced by the "wash and scan" cycles since the likelihood of incorporation of an extra base or failure of incorporation of a base increases during each step (Whiteford et al., 2009). Another limitation of this approach is amplification bias and the template sequence errors contributed by the polymerase chain reaction (PCR) amplification step. Admittedly, NGS has many applications in biological studies, such as DNA-sequencing to assemble a previously unknown genome, and RNA-sequencing to analyze gene expression and to identify the regions of DNA or RNA binding proteins. One of the most important applications of NGS is to identify mutations, including single nucleotide polymorphisms (SNP), small insertions/deletions (INDELs), structural variations, e.g., translocations, inversions, and copy number variations (CNV) (Zhang et al., 2011; Bahassi el and Stambrook, 2014; Wadapurkar and Vyas, 2018).

There are a number of different sequencing approaches that constitute the third generation of sequencing (TGS) paradigm, however, they are primarily distinguished from previous generations in their focus on uninterrupted sequencing of a single DNA or RNA molecule (not an ensemble). This makes them highly preferable for a number of use cases such as de novo assembly, improved genome annotations, and epigenome characterization (Blow et al., 2016; Seo et al., 2016; Jiao et al., 2017). One of the most significant among these approaches is the Single Molecule Real-Time (SMRT) sequencing pioneered by Pacific Biosciences. It uses nanoscale optical waveguide, more specifically zero-mode waveguide (ZMW) technology to be able to directly observe a single DNA polymerase molecule synthesizing a DNA strand. While it is in principle a sequencing by synthesis like Illumina, it does not depend on the "scan and wash" cycles and is therefore able to sequence very long reads largely limited in length by the chemistry of the DNA polymerase and not the underlying technology. As a result, it is possible to get reads of maximum length more than $80 \mathrm{~kb}$ and average length above $20 \mathrm{~kb}$ (Badouin et al., 2017; Jiao and Schneeberger, 2017). Also, it does not suffer from the amplification bias associated with PCR. While it is prone to a higher raw read error rate associated largely with single insertions and deletions, the errors are random (not systematic as in earlier approaches) which can be resolved by the consensus step of the assembly and Illumina short reads polishing. Oxford nanopore sequencing is another approach to single-molecule sequencing (SMS) that has read length, error rate, and throughput similar to PacBio but is primarily available as a portable, cheap, real-time device called MinION that can be directly connected to a computer and conveniently used in the field. It does not depend on chemical labeling of the sample or intervening PCR amplification steps (Ambardar et al., 2016; Rang et al., 2018). Instead, the individual nucleotides are identified as a single DNA or RNA molecule is transported through a nanopore (nanometers in size) using electrophoresis. There also exist a number of other approaches based on the idea of direct imaging of the polynucleotides using tunneling and transmission electron microscopy (Schadt et al., 2010). One of the most important applications of TGS is its role in genome assembly and fulllength transcripts identification due to its ultra long read length compared to NGS. This has resulted in significantly higher quality of genomes for an increasing number of species.

\section{EVOLUTION OF TRANSCRIPT PROFILING}

Some of the earliest attempts at transcript profiling date back to the Sanger sequencing in 1980s, of the expressed sequence tags (ESTs), which are short nucleotide sequences generated from cDNAs (Adams et al., 1991; Marra et al., 1998). Other methods such as Northern blotting and reverse transcriptase quantitative PCR (RT-qPCR) were often used as 
ad hoc options for targeting few transcripts (Alwine et al., 1977; Becker-André and Hahlbrock, 1989; Morozova et al., 2009). The mid-1990s saw the rise of two different genomic scale approaches to transcript characterization, namely serial analysis of gene expression (SAGE) (Velculescu et al., 1995), and DNA microarrays (Lockhart et al., 1996). SAGE involves sequencing (initially Sanger sequencing) of long concatemers of small tags (initially $\sim 10 \mathrm{bp}$ ) that uniquely identify different mRNAs. A statistical analysis of the frequency of the tags and the corresponding mRNA sequences allows a direct transcript quantification and discovery of new genes. Over the years, variations of SAGE have been devised to identify tags more accurately by increasing tag length to 17 (LongSAGE, Saha et al., 2002), 21 (Robust-LongSAGE, Gowda et al., 2004), and 26 (SuperSAGE, Matsumura et al., 2005). Another variation led to massively parallel signature sequencing (MPSS) based on sequencing reads of 16-20 bp (Brenner et al., 2000), which was used to validate the expression of around 10,000 genes in Arabidopsis thaliana (Meyers et al., 2004) and similarly for around 20,000 genes across 32 human tissues (Jongeneel et al., 2005). DNA microarrays (or DNA chips) are based on the concept of measuring the hybridization of the labeled target cDNA strands from sample with the fixed probes (Schena et al., 1995). Because of their high throughput and lower cost, microarrays were widely used throughout 2000s. However, unlike SAGE, they are limited to probing using the array the genes that are already known, so a reference genome or transcriptome is a must for microarrays.

High throughput sequencing, beginning in the early 2000s, has sought to address the limitations inherent to previous approaches. More specifically, RNA-Seq supports both the discovery and quantification of transcripts using a single high-throughput sequencing assay. A reference genome or a transcriptome is used for read alignment but if a reference sequence is not available, a transcriptome can be assembled de novo using the reads and subsequently used for read alignment. Also, it allows quantification of RNAs over a broader dynamic range of five orders of magnitude, as compared to three for microarrays. In addition to gene expression quantification, RNASeq is quite effective in detecting alternative splicing events. As a result, it has grown to be most popular transcript profiling approach over the last decade. However, based on second generation sequencing approaches, the short-read RNA-Seq has several inherent limitations. It fails to accurately identify multiple full-length transcripts reconstituted from the short reads (Steijger et al., 2013; Wang et al., 2016). This problem is pervasive particularly when dealing with complex genomes (mostly eukaryotic), which exhibit a large number of isoforms per gene because of alternative splicing and where genes have multiple candidate promoters and $3^{\prime}$ ends (Conesa et al., 2016). As a result, short-reads RNA-Seq is simply insufficiently equipped in studying gene regulation, the protein-coding potential of the genome and ultimately the phenotypic diversity.

With long-read sequencing technologies, it has become reality that one read is one transcript, and each transcript can be accurately captured and studied individually since it directly provides full-length cDNA sequences (Wang et al., 2016).
Techniques such as Oxford Nanopore and PacBio SMS, are designed to do away with the need to do assembly and therefore are better suited to comprehensively identify full length transcripts and to profile allele specific expression. While TGS techniques are optimal for de novo sequencing for small-tomoderate sized genomes ( $<1 \mathrm{Gbp}$ ), they become cost-prohibitive for high coverage of larger genomes. In such cases, a hybrid approach combining the strengths of SGS and TGS yields less erroneous outcomes at lower costs (Koren et al., 2012; Goodwin et al., 2015; Miller et al., 2017).

Unlike the previous approaches, single-molecule long-read sequencing based transcript profiling techniques have the inherent advantage of rendering, in vitro and without ambiguity, a full-length transcript sequence without depending on the errorprone, computational step of assembly (Abdel-Ghany et al., 2016; Wang et al., 2016; Cheng et al., 2017). As a result, they allow a more precise detection of alternative splicing events and eventually novel isoforms, making it easier to build gene models for species which are poorly studied or have an incomplete or missing reference genome. Next, we will discuss one of the most popular third generation transcript profiling techniques, namely, PacBio Iso-Seq.

\section{PACBIO ISO-SEQ}

Pacific Biosciences offers Iso-Seq protocol for transcript sequencing that includes library construction, cDNA fragment size selection, sequencing, and data analysis for characterization of multiple isoforms (Au et al., 2013; Tilgner et al., 2014; Wang et al., 2016). Here we briefly discuss the in vitro and in silico stages of the Iso-Seq protocol.

\section{Experimental Pipeline}

To get the high-confidence transcripts set, we recommend to start the experimental pipeline with size selection (BluePippin and SageELF ${ }^{\mathrm{TM}}$ Size Selection systems), which will result in libraries for multiple size fractions (e.g., $<1 \mathrm{~kb}, 1-2 \mathrm{~kb}, 2-3 \mathrm{~kb}$, $3-5 \mathrm{~kb}$, and ( $>5 \mathrm{~kb}$ ). Size selection is recommended to get the best out from your libraries since it allows a more accurate detection over a broader range of transcripts. In the absence of size selection, smaller fragments may load preferentially on the sequencer necessitating more SMRT cells in total since each library requires a certain number of cells to get sufficient depth to capture as many transcripts as possible. With the development of sequencing platform and chemistry, it is worth noting that the Sequel sequencing kit and protocols eliminate the need for size selection for transcripts $<4 \mathrm{~kb}$ but size selection can be optionally used to enrich for transcripts $>4 \mathrm{~kb}$ (Figure 1). While this has significantly streamlined the downstream steps in the experimental pipeline, it can potentially introduce sequencing bias for libraries that exhibit a large size range.

\section{Informatics Pipeline}

Next we will discuss the informatics pipeline that leverages the sequencing reads from the experimental pipeline toward the goal of generating high quality isoforms de novo which may 


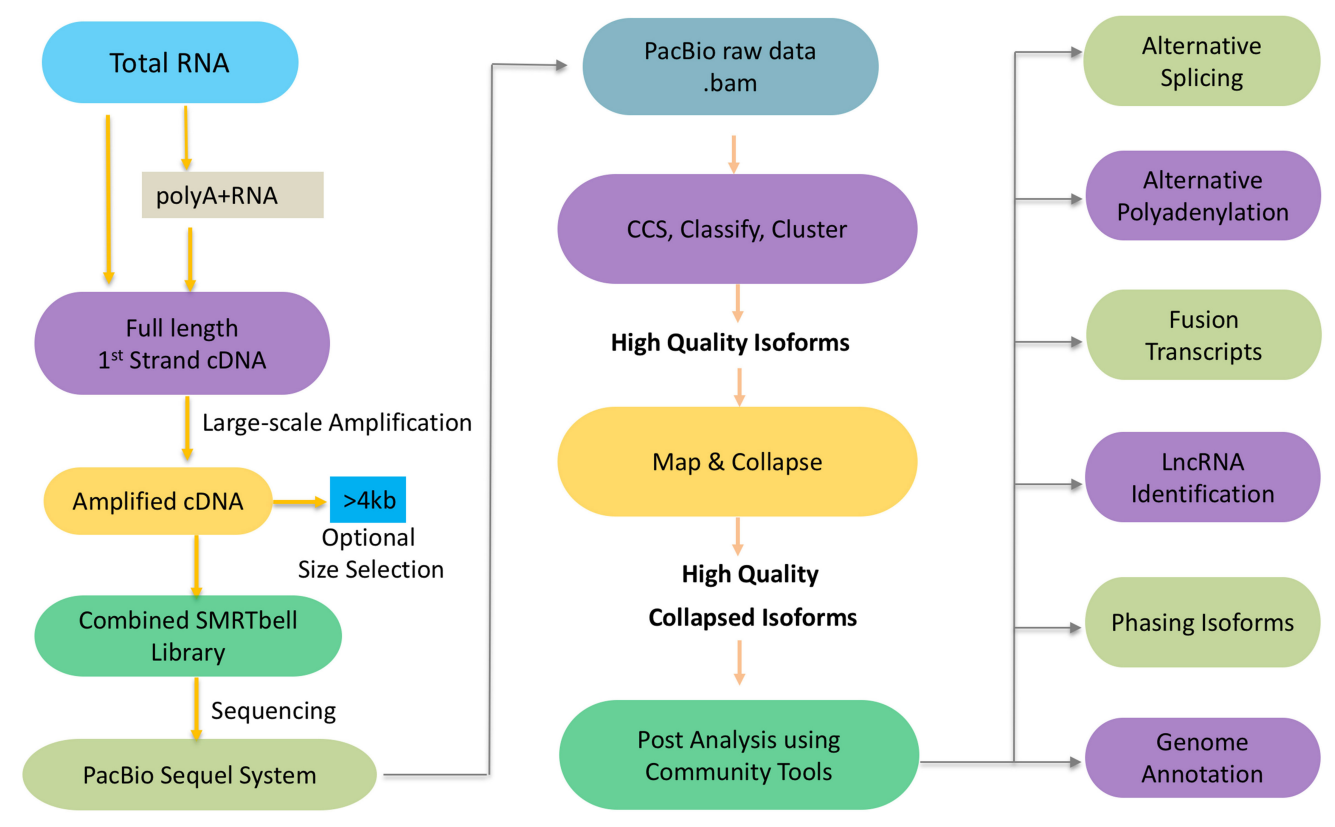

FIGURE 1 | Schematic workflow of Iso-Seq.

optionally be mapped to a reference genome (Figure 1). Here is a brief outline of the steps involved. The PacBio raw reads are continuous long reads (CLR) that need to be trimmed for adapters and filtered for artificial artifacts. Depending on lengths of the CLR and the transcript, the lifetime of the polymerase and the number of times an inserted strand was sequenced (number of passes), one or more subreads are generated. The subreads from a single ZMW are used to generate a circular consensus sequence (CCS) read. The reads are classified into full-length non-chimeric (FLNC), and non-FLNC reads. FLNC reads contain both $5^{\prime}$ and $3^{\prime}$ primers as well as a poly(A) tail preceding the $3^{\prime}$ primer. The FLNC reads are grouped into consensus isoforms using iterative clustering for error (ICE) correction. At this stage, tools such as Quiver (Chin et al., 2013) can be used to incorporate non-FLNC reads to polish the consensus isoforms and select the high quality isoforms. Also, short reads from RNA-Seq if available can be used for an additional step of error correction using tools such as LoRDEC (Salmela and Rivals, 2014), LSC (Au et al., 2012), or Proovread (Hackl et al., 2014). If a reference genome is available, these high quality isoforms can be mapped against it using tools such as GMAP (Wu and Watanabe, 2005), minimap2 (Li, 2018), and STAR (Dobin et al., 2013). The mapped transcripts can be collapsed further to filter out redundant transcripts using ToFU (Gordon et al., 2015) or TAPIS (AbdelGhany et al., 2016). PacBio SMRT Link Suite offers various command line and programmatic options as well as a webbased user interface to support analysis and end-to-end workflow management of the reads from PacBio RS II and Sequel systems (PacBio GitHub ${ }^{1}$ ).

\footnotetext{
${ }^{1}$ https://github.com/PacificBiosciences/pbcommand
}

An improved version of the pipeline, Iso-Seq2, has an extra pre-clustering step to bin full length non-chimeric reads based on gene families. The subsequent steps are similar to Iso-Seq1. The latest version of the pipeline, Iso-Seq3, is designed to scale up to the much higher throughput of Sequel compared to PacBio RS II because of optimization features such as faster clustering algorithms. Also, the Iso-Seq3 pipeline generates relatively fewer but higher quality polished transcripts than Iso-Seq2 because of a more conservative primer removal and barcode demultiplexing step (named, lima). Unlike the previous versions, it also does away the need to use non-full reads. A quality check using SQANTI (Tardaguila et al., 2018) also confirms that Iso-Seq3 generates a higher number of perfectly annotated isoforms. Please see Table 1 for a listing of the Iso-Seq tools discussed in this manuscript.

\section{DOWNSTREAM APPLICATIONS OF ISO-SEQ}

In addition of the discovery of novel transcripts and alternative splicing events, the availability of high quality, full-length isoform sequences greatly impacts our understanding of alternative splicing, alternative polyadenylation (APA), fusion transcripts, long non-coding RNAs (lncRNAs), isoform phasing, and genome annotation (Figure 1).

\section{Identification of Alternative Splicing}

Alternative splicing is one of the most common mechanisms known to increase the diversity of transcripts primarily in eukaryotes. Before the advent of TGS, the traditional method to identify different splicing isoforms has been based on the 
TABLE 1 | List of the Iso-Seq tools along with a brief description of their usage and related online links.

\begin{tabular}{|c|c|c|c|}
\hline Tool & Usage & Website & Literature \\
\hline ASTALAVISTA & Detect alternative splicing events & http://astalavista.sammeth.net/ & $\begin{array}{l}\text { Foissac and Sammeth, } \\
2007\end{array}$ \\
\hline $\mathrm{CASH}$ & Detect alternative splicing events & https://sourceforge.net/projects/cash-program/ & Wu et al., 2018 \\
\hline CodingQuarry & $\begin{array}{l}\text { Gene prediction (HMM-based) using both RNA-Seq } \\
\text { data and genome sequence }\end{array}$ & https://sourceforge.net/projects/codingquarry/ & Testa et al., 2015 \\
\hline GMAP & Spliced alignment to genome & http://research-pub.gene.com/gmap/ & Wu and Watanabe, 2005 \\
\hline LoRDEC & Error correction of FLNC with short read RNA-seq & http://atgc.lirmm.fr/lordec & Salmela and Rivals, 2014 \\
\hline LoReAn & $\begin{array}{l}\text { Comparative analysis and annotation: identify novel } \\
\text { isoforms/genes against reference annotation }\end{array}$ & https://github.com/lfaino/LoReAn & Cook et al., 2018 \\
\hline LSC & Error correction of FLNC with short read RNA-seq & http://augroup.org/LSC/LSC_download.html & Au et al., 2012 \\
\hline minimap2 & Spliced alignment to genome & https://github.com/lh3/minimap2 & Li, 2018 \\
\hline PASA & Detect alternative splicing events & https://pasapipeline.github.io/ & Liu et al., 2017 \\
\hline Proovread & Error correction of FLNC with short read RNA-seq & https://github.com/Biolnf-Wuerzburg/proovread & Hackl et al., 2014 \\
\hline Quiver & Polishing PacBio RS II reads & $\begin{array}{l}\text { https://github.com/PacificBiosciences/ } \\
\text { GenomicConsensus }\end{array}$ & Chin et al., 2013 \\
\hline SpliceGrapher & Detect alternative splicing events & http://splicegrapher.sourceforge.net/ & Rogers et al., 2012 \\
\hline SQANTI & $\begin{array}{l}\text { Comparative analysis and annotation: identify novel } \\
\text { isoforms/genes against reference annotation }\end{array}$ & https://bitbucket.org/ConesaLab/sqanti & Tardaguila et al., 2018 \\
\hline STAR & Spliced alignment to genome & https://github.com/alexdobin/STAR/releases & Dobin et al., 2013 \\
\hline SUPPA & Detect alternative Splicing events & https://bitbucket.org/regulatorygenomicsupf/suppa & Alamancos et al., 2015 \\
\hline TAPIS & $\begin{array}{l}\text { Alternative splicing, collapsing redundant or degraded } \\
\text { transcripts }\end{array}$ & https://bitbucket.org/comp_bio/tapis & Abdel-Ghany et al., 2016 \\
\hline ToFU & Preprocessing (collapse to non-redundant isoforms) & https://github.com/PacificBiosciences/IsoSeq_SA3nUP & Gordon et al., 2015 \\
\hline
\end{tabular}

short-reads sequencing, which assembles short reads into long transcripts based on splice junction reads. This approach often results in prediction of transcripts that do not exist (false positives) or fails to identify true transcripts (false negatives), especially when one gene can transcribe a large number of isoforms. With the development of SMS technology, "one read is one transcript" is not a dream anymore and scientists can get the intact sequence of each isoform by sequencing a single cDNA molecule. Since no assembly is required in this method, it eliminates the assembly errors caused by previous short-reads sequencing and offers particular advantage in characterization of polyploid transcriptomes which have a large number of repeats and homeolog genes. There are a number of different events that can lead to alternative splicing: exon skipping (ES), alternative $5^{\prime}$ splice site (A5), alternative $3^{\prime}$ splice site (A3), mutually exclusive exons (MXE), and intron retention (IR). Tools that detect alternative splicing events include Astalavista (Foissac and Sammeth, 2007), SUPPA (Alamancos et al., 2015), PASA (Program to Assemble Spliced Alignments) (Liu et al., 2017), SpliceGrapher (Rogers et al., 2012), and CASH (Wu et al., 2018). Compared to SGS based tools such as reference-guided (Cufflinks, StringTie) or de novo (Trinity, Oases, Velvet), Iso-Seq is known to retrieve longer isoforms as well as more number of isoforms (both total and per gene) (Gordon et al., 2015; Wang et al., 2016). This has revolutionized our understanding of the biology of a number of organisms, including plants and animals since transcript diversity usually represents functional diversity, indicating the potential important biological functions of these novel identified isoforms (Au et al., 2013; Abdel-Ghany et al., 2016; Wang et al., 2016, 2018; Kuo et al., 2017).

\section{Identification of Alternative Polyadenylation}

In addition to the APA is another widespread mechanism in complex genomes, particularly eukaryotic, for posttranscriptional regulation of function, stability, localization, and translation efficiency (Shen et al., 2008, 2011). Alternative polyadenylation controls gene expression by virtue of selection of alternate $\operatorname{poly}(\mathrm{A})$ sites in the $3^{\prime}$ end of the pre-mRNA, thus letting a gene encode multiple mRNA transcripts which vary in their coding sequence (CDS) or often in their $3^{\prime} \mathrm{UTR}$ regions. While normally, it is found in the distal region of $3^{\prime}$ UTR, it has number of other variations including proximal region of $3^{\prime}$ UTR, alternative terminal exons, intronic sites, and exonic CDS sites (Gruber et al., 2013). Once again, while it's challenging to detect alternative polyadenylation sites using short reads from SGS, full-length cDNA sequencing from Iso-Seq is able to detect genome-wide alternative polyadenylation sites, and the $3^{\prime}$ end is more accurate because of the poly $(\mathrm{A})$ selection during the library construction. As a result, alternative polyadenylation motif has been identified from different species (Abdel-Ghany et al., 2016; Wang et al., 2018).

\section{Fusion Transcript Identification}

A fusion transcript is a chimeric RNA made of two or more transcripts. Often, the constituent transcripts correspond to two distinct genes brought together into a fusion gene at DNA level because of translocation, interstitial deletion, or inversion. Alternatively, transcripts can fuse at RNA level by the trans-splicing or cis-splicing between the neighboring genes (Kumar et al., 2016). The constituent transcripts must map to 


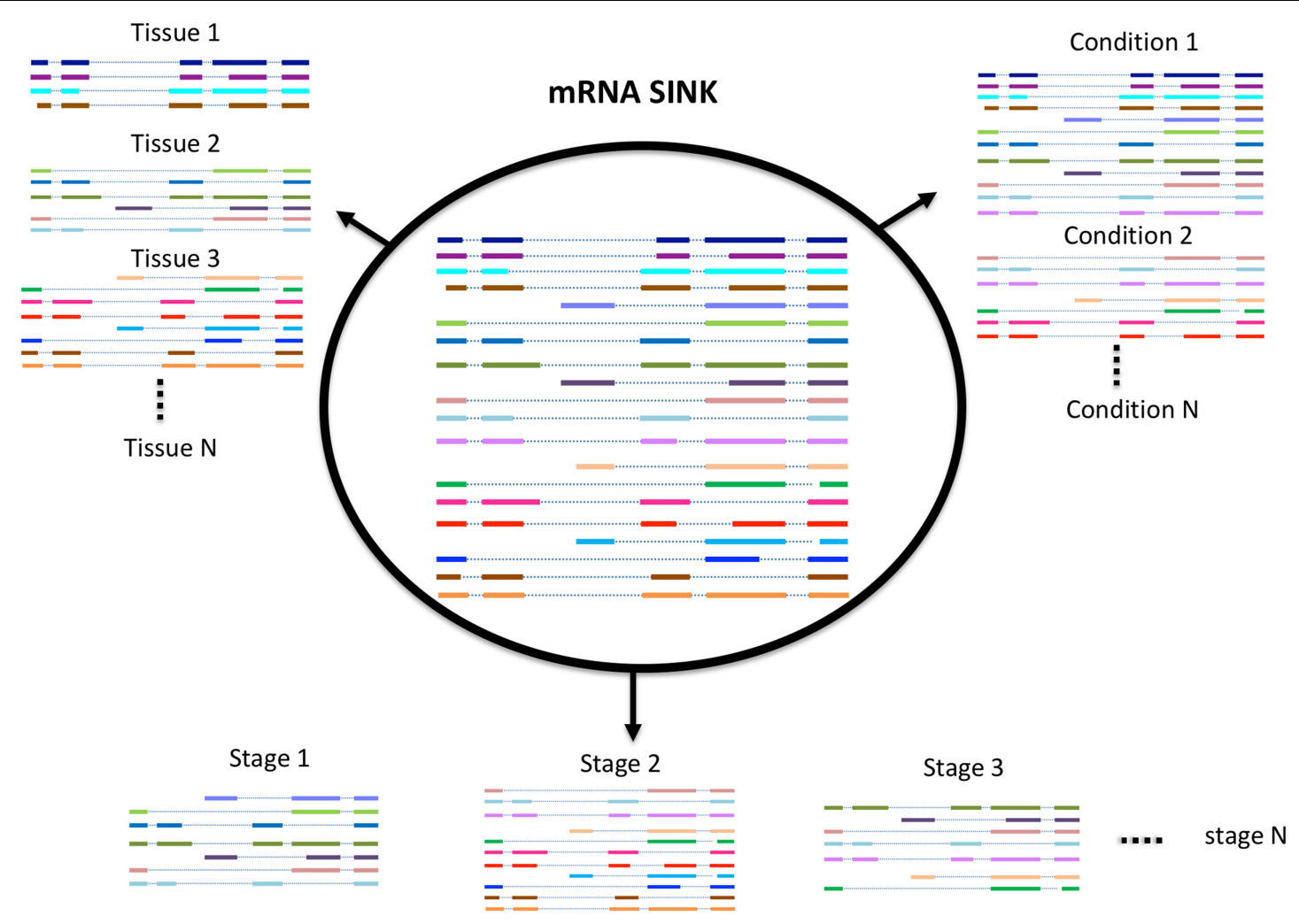

FIGURE 2 | Schematic model of alternative splicing utilization.

two or more loci which are at least $100 \mathrm{~kb}$ apart, align at least $10 \%$ with the corresponding transcripts and together contribute to at least $99 \%$ alignment coverage (Wang et al., 2016). While there exist dozens of SGS tools that can detect fusion transcripts, they are limited because of mapping errors inherent to short reads and the assembly. The Cupcake ToFu (Gordon et al., 2015) developed by PacBio has been able to identify candidate fusion transcripts, and another tool is Isoform Detection and Prediction (IDP-fusion) which uses a hybrid approach based on SGS and TGS reads and was able to identify fusion genes and their isoforms in cancer transcriptomes (Weirather et al., 2015). However, those candidate fusion transcripts usually have high false positive rate which need further validation through different approaches, e.g., RT-PCR followed by Sanger sequencing or single-molecule mRNA Fluorescent in situ Hybridization (RNA FISH) (Semrau et al., 2014; Wang et al., 2016).

\section{Single-Molecule Sequencing Facilitates Genome Annotation}

Many of the commonly used annotation pipelines use a combination of $a b$ initio and evidence based predictions to generate accurate consensus annotations. MAKER2 is a user-friendly, fully automated annotation pipeline that incorporates multiple sources of gene prediction information and has been extensively used to annotate eukaryotic genomes (Holt and Yandell, 2011). The Broad Institute Eukaryotic
Genome Annotation Pipeline (Haas et al., 2011) has mainly been used to annotate fungal genomes and integrates multiple programs and evidences for genome annotation. CodingQuarry (Testa et al., 2015) is another gene prediction software that utilizes general hidden Markov models for gene prediction using both RNA-Seq data and genome sequence. However, most of these tools are not designed to exploit gene structure information from single-molecule cDNA sequencing.

The use of single-molecule cDNA sequencing can increase the accuracy of automated genome annotation by improving genome mapping of sequencing data, correctly identifying intron exon boundaries, directly identifying alternatively spliced transcripts, identifying transcription start and end sites, and providing precise strand orientation to single exons genes. The full-length transcripts mapped against a reference genome can be used to improve or add de novo structural and functional annotation to a genome, improve genome assembly and existing gene models. Previous studies have demonstrated the advantage of SMS by discovering longer and novel transcripts/genes, lncRNAs, and even fusion transcripts as well (Abdel-Ghany et al., 2016; Wang et al., 2016). To address the disconnection between genome annotations and the latest sequencing technologies, recently, the Long Read Annotation (LoReAn) pipeline has been developed (Cook et al., 2018). LoReAn is an automated annotation pipeline that takes full advantage of MinION or PacBio SMRT long-read sequencing 


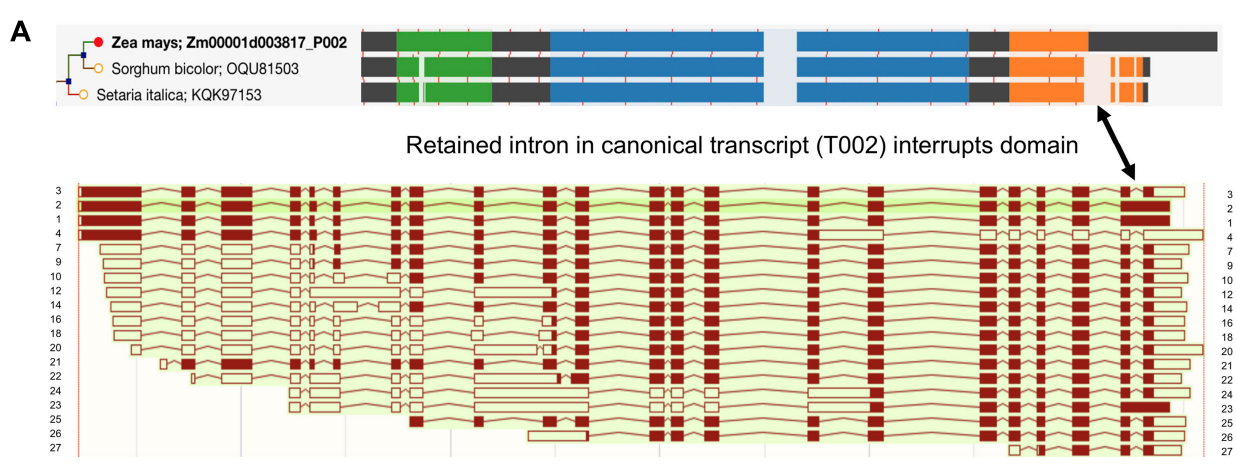

B

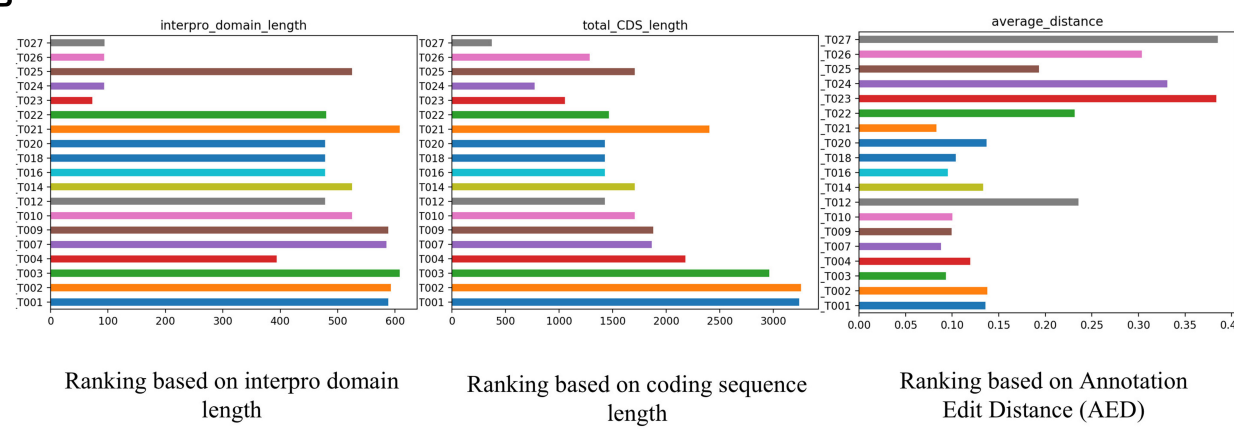

FIGURE 3 | Alignment and ranking of different isoforms. (A) Gene tree multiple sequence alignment color coded by interpro domain. The orange domain is conserved in grass orthologs, but is not identified completely in maize because a retained intron disrupts it and induces a frame shift (shown by arrow). The resulting longer translation was selected for analysis in the gene tree pipeline. Different colors mean different interpro domains, black color means "no domain here," lightly shaded area indicates a gap in the multiple sequence alignment. The thin red lines show the positions of exon junctions. (B) Ranking of different isoforms of Zm00001d003817 based on different standards. T002 has the longest CDS, but T003 outperforms it in domain length and annotation edit distance.

data in combination with protein evidence and $a b$ initio gene predictions for full genome annotation. Short-read RNASeq can be used in LoReAn to train $a b$ initio software. Based on the reannotation of two fungal and two plant species, LoReAn has been shown to provide annotations with increased accuracy by incorporating single-molecule cDNA sequencing data from different sequencing platforms. SQANTI (Tardaguila et al., 2018) is another pipeline for structural and quality annotation of novel transcript isoforms. It takes as input the full length transcripts and a reference genome and associated annotations, and provides a deep characterization of isoforms at both transcript and junction level. It generates gene models and classifies transcripts based on splice junctions and donor and acceptor sites. In addition, it can also filter out isoforms that are likely to be artifacts.

\section{Single-Molecule Sequencing Enables Isoform Phasing}

Haplotype phasing of genetic variants is important for interpretation of the genome, population genetic analysis, and functional genomic analysis of allelic activity. Even though more and more long-read sequencing reads have been generated for different studies, there is not much investigation on the allelic variants so far. Such information is crucial for understanding allelic transcriptomes, the parent origin of each allele, and their potential biological consequences. SMS has been used successfully to identify full-length gene isoforms and thus have the potential to overcome the haplotyping problem due to its multi-kilobase reads length. Recently, a series of tools have been developed for the haplotyping of single-molecule isoforms. IDP-ASE was developed for haplotyping and quantification of Allele-specific expression (ASE) at both the gene and isoform levels requiring only RNA sequencing data (Deonovic et al., 2017). HapIso is another method for the reconstruction of the haplotype specific isoforms of a diploid cell, which is able to tolerate the relatively high error-rate of the SMS and discriminate the reads into the paternal alleles of the isoform transcript (Mangul et al., 2017). phASER (Castel et al., 2016), was developed to incorporate RNA-Seq and DNA-Seq data with population phasing, allowing phasing over longer distances. And IsoPhase, which is under development from PacBio, is designed to phase the isoforms from diploid or even tetraploid organisms. With IsoPhase, parent-of-origin allele specific isoforms can be identified in the hybrids. Firstly CCS reads are aligned to genome, then individual SNPs are called, and full length reads are used to infer haplotypes, residual sequencing errors are corrected to get to the number of 
expected alleles, finally the number of full-length reads of each haplotype can be called.

\section{SPATIO-TEMPORAL VARIABILITY IN TRANSCRIPTOME PROFILE}

While Iso-Seq has been successful in identifying a large number of novel and longer transcripts in almost all species where it was used, most of these transcripts lack an experimental or evidence-based functional characterization. A number of studies exist that have demonstrated that the number of transcripts expressed in an organism (transcriptome profile) depends on many factors such as environmental stress, growth condition, developmental stage, and tissue type (Figure 2; Wang et al., 2016, 2018; Zhu F.Y. et al., 2017). Therefore, the diversity of transcripts in one organism can be increased with the sequencing of more and more tissues. Previous approaches mostly use short reads sequencing to identify potential transcripts in a certain tissue, this approach is good to quantify the expression level of each transcript, but not able to give the accurate information or complete structure of the transcript. In contrast, SMS due to its ultra long reads methodology is significantly more accurate. Recently, it has also become feasible to study the full-length transcriptome at single cell level both in animals and in plants (Zhu S. et al., 2017; Ryu et al., 2019). We believe with the development of new techniques and participation from more labs, the diversity of transcriptome within or between species will be further revealed.

\section{HOW TO DEAL WITH MULTIPLE ISOFORMS IDENTIFIED FROM ISO-SEQ?}

While single-molecule long read sequencing based approaches have identified a wide array of novel transcripts which were generated from different splicing patterns (Figure 3A), they need to be validated and characterized since not all of them have a meaningful impact on the cellular biological processes of the cell. Recent studies in maize and sorghum (Wang et al., 2018) showed that $\sim 45 \%$ of the isoforms could undergo Non-Sense Mediated Decay (NMD) after mRNA processing; that being said, a large number of the transcripts potentially will be degraded before transportation to the cell and the rest of transcripts are more likely to have biological functions. Therefore, there is clearly a need to be able to judge the validity and usage of these isoforms. We propose that high confidence transcripts can be ranked for validity based on criteria such as open reading frame $(\mathrm{ORF})$ and $\mathrm{CDS}$ length, Interpro domain coverage, annotation edit distance, and their spatio-temporal expression levels. Figure 3B illustrates the application of such criteria to an

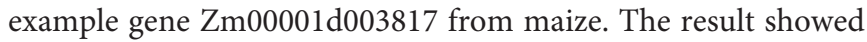
that the ranking of isoforms can be different using different criteria. Due to an IR in T002 isoform, its ORF was shifted, and as a result the protein domain which is conserved in grass orthologs is not completely identified in maize. T002 has the longest CDS, but T003 outperforms it in domain length and annotation edit distance.

\section{COMPARATIVE SINGLE-MOLECULE TRANSCRIPTOME STUDIES BETWEEN CLOSE SPECIES, WHAT TO COMPARE?}

A growing number of SMS based transcriptome studies have made it possible to compare full-length transcriptomes between evolutionarily close species and identify the cause of divergence of different phenotypes between species. Based on the orthologous genes in the two organisms and the associated full-length transcriptomes, we can now compare the splicing variants between species and better understand the conservation of genes/isoforms, the divergence of splicing patterns, and the significance of their expression levels. The first SMS based comparative transcriptome study was performed between maize and sorghum by Wang et al. (2018). Conserved genes and isoforms were identified between these two species, gene expression and alternative splicing were found to be playing an important role in the plant architecture divergence between evolutionarily close species. However, certain requirements are needed to perform these comparative studies, such as: (1) tissues selected in this study should be at same or very similar developmental stage for the comparison to be meaningful; (2) there should exist a threshold depth of sequencing, so that majority of isoforms will be captured in each tissue/organism.

\section{CONCLUSION}

It is worth noting that as the TGS platforms continue to mature, they are not without their own set of challenges. Three of the more common challenges associated especially with the early PacBio long reads are the raw read errors, low throughput and high cost. Higher than acceptable errors in raw reads limit the de novo transcript identifications, necessitating the need for the reference genome (Au et al., 2012). Low throughput from SMRT cells makes it difficult to accurately quantify the transcript expression. As a result, most of the captured isoforms are highly expressed isoforms and the lowly expressed isoforms are usually lost. Also relatively longer transcripts are more likely to be missed due to longer polymerase lifetime required to allow fulllength pass during the sequencing. That being said, sequencing depth matters for Iso-Seq study, especially when it comes to comparison between different tissues or conditions, or even different species, therefore a higher sequencing depth is necessary to make the comparison convincing. As a gap-fill measure, the long read dataset can be supplemented with more accurate and abundant short reads, if available, to address these issues (Hansen et al., 2011). PacBio Sequel, with its improved chemistry, tries to address these concerns by offering higher sequencing lengths amenable to more number of passes for consensus autocorrection as well as higher throughput from SMRT cells.

With the reality that Iso-Seq transcripts have been used to annotate more and more genomes, another challenge is the 
need to rank and prioritize for community research the growing number of isoforms identified from different tissues/conditions within an organism. While SMS has dominated the transcriptome sequencing with its power of identification of full-length information of each transcript, it has raised new questions such as, how to deal with the large number of newly identified isoforms and what are their functions. Experimental approaches such as CRISPR could help by targeting the role of each isoform, and see if there are redundant or complementary functions among these different splicing isoforms.

\section{REFERENCES}

Abdel-Ghany, S. E., Hamilton, M., Jacobi, J. L., Ngam, P., Devitt, N., Schilkey, F., et al. (2016). A survey of the sorghum transcriptome using singlemolecule long reads. Nat. Commun. 2016:11706. doi: 10.1038/ncomms1 1706

Adams, M. D., Kelley, J. M., Gocayne, J. D., Dubnick, M., Polymeropoulos, M. H., Xiao, H., et al. (1991). Complementary DNA sequencing: expressed sequence tags and human genome project. Science 252, 1651-1656.

Alamancos, G. P., Pagès, A., Trincado, J. L., Bellora, N., and Eyras, E. (2015). Leveraging transcript quantification for fast computation of alternative splicing profiles. RNA 21, 1521-1531. doi: 10.1261/rna.051557.115

Alwine, J. C., Kemp, D. J., and Stark, G. R. (1977). Method for detection of specific RNAs in agarose gels by transfer to diazobenzyloxymethyl-paper and hybridization with DNA probes. Proc. Natl. Acad. Sci. U.S.A. 74, 5350-5354.

Ambardar, S., Gupta, R., Trakroo, D., Lal, R., and Vakhlu, J. (2016). High throughput sequencing: an overview of sequencing chemistry. Indian J. Microbiol. 56, 394-404.

Ardui, S., Ameur, A., Vermeesch, J. R., and Hestand, M. S. (2018). Single molecule real-time (SMRT) sequencing comes of age: applications and utilities for medical diagnostics. Nucleic Acids Res. 46, 2159-2168. doi: 10.1093/nar/gky066

Au, K. F., Sebastiano, V., Afshar, P. T., Durruthy, J. D., Lee, L., Williams, B. A., et al. (2013). Characterization of the human ESC transcriptome by hybrid sequencing. Proc. Natl. Acad. Sci. U.S.A. 110, E4821-E4830. doi: 10.1073/pnas. 1320101110

Au, K. F., Underwood, J. G., Lee, L., and Wong, W. H. (2012). Improving PacBio long read accuracy by short read alignment. PLoS One 7:e46679. doi: 10.1371/ journal.pone.0046679

Badouin, H., Gouzy, J., Grassa, C. J., Murat, F., Staton, S. E., Cottret, L., et al. (2017). The sunflower genome provides insights into oil metabolism, flowering and Asterid evolution. Nature 546, 148-152. doi: 10.1038/nature22380

Bahassi el, M., and Stambrook, P. J. (2014). Next-generation sequencing technologies: breaking the sound barrier of human genetics. Mutagenesis 29, 303-310. doi: 10.1093/mutage/geu031

Becker-André, M., and Hahlbrock, K. (1989). Absolute mRNA quantification using the polymerase chain reaction (PCR). A novel approach by a PCR aided transcript titration assay (PATTY). Nucleic Acids Res. 17, 9437-9446.

Blow, M. J., Clark, T. A., Daum, C. G., Deutschbauer, A. M., Fomenkov, A., Fries, R., et al. (2016). The epigenomic landscape of prokaryotes. PLoS Genet. 12:e1005854. doi: 10.1371/journal.pgen.1005854

Brenner, S., Johnson, M., Bridgham, J., Golda, G., Lloyd, D. H., Johnson, D., et al. (2000). Gene expression analysis by massively parallel signature sequencing (MPSS) on microbead arrays. Nat. Biotechnol. 18, 630-634.

Casamassimi, A., Federico, A., Rienzo, M., Esposito, S., and Ciccodicola, A. (2017). Transcriptome profiling in human diseases: new advances and perspectives. Int. J. Mol. Sci. 18:E1652. doi: 10.3390/ijms18081652

Castel, S. E., Mohammadi, P., Chung, W. K., Shen, Y., and Lappalainen, T. (2016). Rare variant phasing and haplotypic expression from RNA sequencing with phASER. Nat. Commun. 7:12817. doi: 10.1038/ncomms12817

Cheng, B., Furtado, A., and Henry, R. J. (2017). Long-read sequencing of the coffee bean transcriptome reveals the diversity of full-length transcripts. Gigascience 6, 1-13. doi: 10.1093/gigascience/gix086

\section{AUTHOR CONTRIBUTIONS}

BW and VK developed the conceptual outline and drafted the manuscript. BW, VK and AO contributed figures and a table. All authors contributed to reviewing the final manuscript.

\section{FUNDING}

This work was supported by USDA 8062-21000-044-00D and NSF IOS 1127112.

Chin, C. S., Alexander, D. H., Marks, P., Klammer, A. A., Drake, J., Heiner, C., et al. (2013). Nonhybrid, finished microbial genome assemblies from longread SMRT sequencing data. Nat. Methods 10, 563-569. doi: 10.1038/nmeth. 2474

Conesa, A., Madrigal, P., Tarazona, S., Gomez-Cabrero, D., Cervera, A., McPherson, A., et al. (2016). A survey of best practices for RNA-seq data analysis. Genome Biol. 17:13. doi: 10.1186/s13059-016-0881-8

Cook, D., Valle-Inclan, J. E., Pajoro, A., Rovenich, H., Thomma, B., and Faino, L. (2018). Long read annotation (LoReAn): automated eukaryotic genome annotation based on long-read cDNA sequencing. Plant Physiol. 179, 38-54. doi: 10.1104/pp.18.00848

Deonovic, B., Wang, Y., Weirather, J., Wang, X. J., and Au, K. F. (2017). IDPASE: haplotyping and quantifying allele-specific expression at the gene and gene isoform level by hybrid sequencing. Nucleic Acids Res. 45:e32. doi: 10.1093/nar/ gkw1076

Dobin, A., Davis, C. A., Schlesinger, F., Drenkow, J., Zaleski, C., Jha, S., et al. (2013). STAR: ultrafast universal RNA-seq aligner. Bioinformatics 29, 15-21. doi: 10.1093/bioinformatics/bts635

Foissac, S., and Sammeth, M. (2007). Astalavista: dynamic and flexible analysis of alternative splicing events in custom gene datasets. Nucleic Acids Res. 35, 297-299.

Goodwin, S., Gurtowski, J., Ethe-Sayers, S., Deshpande, P., Schatz, M. C., and McCombie, W. R. (2015). Oxford Nanopore sequencing, hybrid error correction, and de novo assembly of a eukaryotic genome. Genome Res. 25, 1750-1756. doi: 10.1101/gr.191395.115

Goodwin, S., McPherson, J. D., and McCombie, W. R. (2016). Coming of age: ten years of next-generation sequencing technologies. Nat. Rev. Genet. 17, 333-351. doi: 10.1038/nrg.2016.49

Gordon, S. P., Tseng, E., Salamov, A., Zhang, J., Meng, X., Zhao, Z., et al. (2015). Widespread polycistronic transcripts in fungi revealed by single-molecule mRNA sequencing. PLoS One 10:e0132628. doi: 10.1371/journal.pone.0132628

Gowda, M., Jantasuriyarat, C., Dean, R. A., and Wang, G. L. (2004). RobustLongSAGE (RL-SAGE): a substantially improved LongSAGE method for gene discovery and transcriptome analysis. Plant Physiol. 134, 890-897. doi: 10.1104/ pp.103.034496

Gruber, A. R., Martin, G., Keller, W., and Zavolan, M. (2013). Means to an end: mechanisms of alternative polyadenylation of messenger RNA precursors. Wiley Interdiscip. Rev. RNA 5, 183-196. doi: 10.1002/wrna.1206

Haas, B. J., Zeng, Q., Pearson, M. D., Cuomo, C., and Wortman, J. R. (2011). Approaches to fungal genome annotation. Mycology 2, 118-141. doi: 10.1080/ 21501203.2011.606851

Hackl, T., Hedrich, R., Schultz, J., and Förster, F. (2014). proovread : largescale high-accuracy PacBio correction through iterative short read consensus. Bioinformatics 30, 3004-3011. doi: 10.1093/bioinformatics/btu392

Hansen, K. D., Wu, Z., Irizarry, R. A., and Leek, J. T. (2011). Sequencing technology does not eliminate biological variability. Nat. Biotechnol. 29, 572-573.

Heather, J. M., and Chain, B. (2016). The sequence of sequencers: the history of sequencing DNA. Genomics 107, 1-8. doi: 10.1016/j.ygeno.2015. 11.003

Holt, C., and Yandell, M. (2011). MAKER2: an annotation pipeline and genomedatabase management tool for second-generation genome projects. BMC Bioinformatics 12:491. doi: 10.1186/1471-2105-12-491 
Jain, M. (2012). Next-generation sequencing technologies for gene expression profiling in plants. Brief. Funct. Genomics 11, 63-70. doi: 10.1093/bfgp/elr038

Jiao, W. B., and Schneeberger, K. (2017). The impact of third generation genomic technologies on plant genome assembly. Curr. Opin. Plant Biol. 36, 64-70. doi: 10.1016/j.pbi.2017.02.002

Jiao, Y. P., Peluso, P., Shi, J. H., Liang, T., Stitzer, M. C., Wang, B., et al. (2017). Improved maize reference genome with single-molecule technologies. Nature 546, 524-527. doi: 10.1038/nature22971

Jongeneel, C. V., Delorenzi, M., Iseli, C., Zhou, D., Haudenschild, C. D., Khrebtukova, I., et al. (2005). An atlas of human gene expression from massively parallel signature sequencing (MPSS). Genome Res. 15, 1007-1014. doi: 10.1101/gr.4041005

Karsch-Mizrachi, I., Takagi, T., Cochrane, G., and International Nucleotide Sequence Database Collaboration (2018). The international nucleotide sequence database collaboration. Nucleic Acids Res. 46, D48-D51. doi: 10.1093/ nar/gkx1097

Koren, S., Schatz, M. C., Walenz, B. P., Martin, J., Howard, J. T., Ganapathy, G., et al. (2012). Hybrid error correction and de novo assembly of singlemolecule sequencing reads. Nat. Biotechnol. 30, 693-700. doi: 10.1038/nbt. 2280

Kumar, S., Razzaq, S. K., Vo, A. D., Gautam, M., and Li, H. (2016). Identifying fusion transcripts using next generation sequencing. Wiley Interdiscip. Rev. RNA 7, 811-823. doi: 10.1002/wrna.1382

Kuo, R. I., Tseng, E., Eory, L., Paton, I. R., Archibald, A. L., and Burt, D. W. (2017). Normalized long read RNA sequencing in chicken reveals transcriptome complexity similar to human. BMC Genomics 18:323. doi: 10.1186/s12864-017$3691-9$

Lathe, W., Williams, J., Mangan, M., and Karolchik, D. (2008). Genomic data resources: challenges and promises. Nat. Educ. 1:2.

Levy, S. E., and Myers, R. M. (2016). Advancements in next-generation sequencing. Annu. Rev. Genomics Hum. Genet. 17, 95-115. doi: 10.1146/annurev-genom083115-022413

Li, H. (2018). Minimap2: pairwise alignment for nucleotide sequences. Bioinformatics 34, 3094-3100. doi: 10.1093/bioinformatics/bty191

Liu, X., Mei, W., Soltis, P. S., Soltis, D. E., and Barbazuk, W. B. (2017). Detecting alternatively spliced transcript isoforms from single-molecule longread sequences without a reference genome. Mol. Ecol. Resour. 17, 1243-1256. doi: 10.1111/1755-0998.12670

Lockhart, D. J., Dong, H., Byrne, M. C., Follettie, M. T., Gallo, M. V., Chee, M. S., et al. (1996). Expression monitoring by hybridization to high-density oligonucleotide arrays. Nat. Biotechnol. 14, 1675-1680.

Lowe, R., Shirley, N., Bleackley, M., Dolan, S., and Shafee, T. (2017). Transcriptomics technologies. PLoS Comput. Biol. 13:e1005457. doi: 10.1371/ journal.pcbi.1005457

Mangul, S., Yang, T. H., Hormozdiari, F., Dainis, A. M., Tseng, E., Ashley, E. A., et al. (2017). HapIso: an accurate method for the haplotype- specific isoforms reconstruction from long single-molecule reads. IEEE Trans. Nanobiosci. 16, 108-115. doi: 10.1109/TNB.2017.2675981

Marra, M. A., Hillier, L., and Waterston, R. H. (1998). Expressed sequence tags: ESTablishing bridges between genomes. Trends Genet. 14, 4-7.

Matsumura, H., Ito, A., Saitoh, H., Winter, P., Kahl, G., Reuter, M., et al. (2005). SuperSAGE. Cell Microbiol. 7, 11-18. doi: 10.1111/j.1462-5822.2004.00478.x

Maxam, A. M., and Gilbert, W. (1977). A new method for sequencing DNA. Proc. Natl. Acad. Sci. U.S.A. 74, 560-564. doi: 10.1073/pnas.74.2.560

Meyers, B. C., Vu, T. H., Tej, S. S., Ghazal, H., Matvienko, M., Agrawal, V., et al. (2004). Analysis of the transcriptional complexity of Arabidopsis thaliana by massively parallel signature sequencing. Nat. Biotechnol. 22, 1006-1011. doi: $10.1038 /$ nbt992

Miller, J. R., Zhou, P., Mudge, J., Gurtowski, J., Lee, H., Ramaraj, T., et al. (2017). Hybrid assembly with long and short reads improves discovery of gene family expansions. BMC Genomics 18:541. doi: 10.1186/s12864-017-3 927-8

Moorthie, S., Mattocks, C. J., and Wright, C. F. (2011). Review of massively parallel DNA sequencing technologies. Hugo J. 5, 1-12.

Morozova, O., Hirst, M., and Marra, M. A. (2009). Applications of new sequencing technologies for transcriptome analysis. Annu. Rev. Genomics Hum. Genet. 10, 135-151. doi: 10.1146/annurev-genom-082908-145957
Rang, F. J., Kloosterman, W. P., and de Ridder, J. (2018). From squiggle to basepair: computational approaches for improving nanopore sequencing read accuracy. Genome Biol. 13:90. doi: 10.1186/s13059-018-1462-9

Rogers, M. F., Thomas, J., Reddy, A. S., and Ben-Hur, A. (2012). Splicegrapher: detecting patterns of alternative splicing from RNA-SEQ data in the context of gene models and EST data. Genome Biol. 13:R4. doi: 10.1186/gb-2012-13-1-r4

Ryu, K. H., Huang, L., Kang, H. M., and Schiefelbein, J. (2019). Single-cell RNA sequencing resolves molecular relationships among individual plant cells. Plant Physiol. 179, 1444-1456. doi: 10.1104/pp.18.01482

Saccone, C., and Pesole, G. (2003). Handbook of Comparative Genomics: Principles and Methodology. New York, NY: Wiley-Liss, 133.

Saha, S., Sparks, A. B., Rago, C., Akmaev, V., Wang, C. J., Vogelstein, B., et al. (2002). Using the transcriptome to annotate the genome. Nat. Biotechnol. 20, 508-512. doi: $10.1038 / n b t 0502-508$

Salmela, L., and Rivals, E. (2014). LoRDEC: accurate and efficient long read error correction. Bioinformatics 30, 3506-3514. doi: 10.1093/bioinformatics/btu538

Sanger, F., and Coulson, A. R. (1975). A rapid method for determining sequences in DNA by primed synthesis with DNA polymerase. J. Mol. Biol. 94, 441-448.

Sanger, F., Nicklen, S., and Coulson, A. R. (1977). DNA sequencing with chainterminating inhibitors. Proc. Natl. Acad. Sci. U.S.A. 74, 5463-5467.

Schadt, E. E., Turner, S., and Kasarskis, A. (2010). A window into third-generation sequencing. Hum. Mol. Genet. 19, 227-240. doi: 10.1093/hmg/ddq416

Schena, M., Shalon, D., Davis, R. W., and Brown, P. O. (1995). Quantitative monitoring of gene expression patterns with a complementary DNA microarray. Science 270, 467-470.

Schloss, J. A. (2008). How to get genomes at one ten-thousandth the cost. Nat. Biotechnol. 26, 1113-1115.

Semrau, S., Crosetto, N., Bienko, M., Boni, M., Bernasconi, P., Chiarle, R., et al. (2014). FuseFISH: robust detection of transcribed gene fusions in single cells. Cell Rep. 6, 18-23. doi: 10.1016/j.celrep.2013.12.002

Seo, J. S., Rhie, A., Kim, J., Lee, S., Sohn, M. H., Kim, C. U., et al. (2016). Denovo assembly and phasing of a Korean human genome. Nature 538, 243-247. doi: $10.1038 /$ nature20098

Shen, Y., Ji, G., Haas, B. J., Wu, X., Zheng, J., Reese, G. J., et al. (2008). Genome level analysis of rice mRNA 3 '-end processing signals and alternative polyadenylation. Nucleic Acids Res. 36, 3150-3161. doi: 10.1093/nar/gkn158

Shen, Y., Venu, R. C., Nobuta, K., Wu, X., Notibala, V., Demirci, C., et al. (2011). Transcriptome dynamics through alternative polyadenylation in developmental and environmental responses in plants revealed by deep sequencing. Genome Res. 21, 1478-1486. doi: 10.1101/gr.114744.110

Shendure, J., Balasubramanian, S., Church, G. M., Gilbert, W., Rogers, J., Schloss, J. A., et al. (2017). DNA sequencing at 40: past, present and future. Nature 550, 345-353. doi: 10.1038/nature24286

Steijger, T., Abril, J. F., Engström, P. G., Kokocinski, F., Rgasp Consortium, Hubbard, T. J., et al. (2013). Assessment of transcript reconstruction methods for RNA-seq. Nat. Methods 10, 1177-1184. doi: 10.1038/nmeth.2714

Tardaguila, M., de la Fuente, L., Marti, C., Pereira, C., Pardo-Palacios, F. J., Del Risco, H., et al. (2018). SQANTI: extensive characterization of long-read transcript sequences for quality control in full-length transcriptome identification and quantification. Genome Res. 28, 396-411. doi: 10.1101/gr.222976.117

Testa, A. C., Hane, J. K., Ellwood, S. R., and Oliver, R. P. (2015). CodingQuarry: highly accurate hidden Markov model gene prediction in fungal genomes using RNA-seq transcripts. BMC Genomics 16:170. doi: 10.1186/s12864-015-1344-4

Tilgner, H., Grubert, F., Sharon, D., and Snyder, M. P. (2014). Defining a personal, allele-specific, and single-molecule long-read transcriptome. Proc. Natl. Acad. Sci. U.S.A. 111, 9869-9874. doi: 10.1073/pnas.1400447111

Velculescu, V. E., Zhang, L., Vogelstein, B., and Kinzler, K. W. (1995). Serial analysis of gene expression. Science 270, 484-487. doi: 10.1126/science.270. 5235.484

Wadapurkar, R. M., and Vyas, R. (2018). Computational analysis of next generation sequencing data and its applications in clinical oncology. Inform. Med. Unlocked 11, 75-82.

Wang, B., Regulski, M., Tseng, E., Olson, A., Goodwin, S., McCombie, W. R., et al. (2018). A comparative transcriptional landscape of maize and sorghum obtained by single-molecule sequencing. Genome Res. 28, 921-932. doi: 10. 1101/gr.227462.117 
Wang, B., Tseng, E., Regulski, M., Clark, T. A., Hon, T., Jiao, Y., et al. (2016). Unveiling the complexity of the maize transcriptome by singlemolecule long-read sequencing. Nat. Commun. 7:11708. doi: 10.1038/ncomms 11708

Weirather, J. L., Afshar, P. T., Clark, T. A., Tseng, E., Powers, L. S., Underwood, J. G., et al. (2015). Characterization of fusion genes and the significantly expressed fusion isoforms in breast cancer by hybrid sequencing. Nucleic Acids Res. 43:e116. doi: 10.1093/nar/gkv562

Whiteford, N., Skelly, T., Curtis, C., Ritchie, M. E., Lohr, A., Zaranek, A. W., et al. (2009). Swift: primary data analysis for the Illumina Solexa sequencing platform. Bioinformatics 25, 2194-2199. doi: 10.1093/bioinformatics/btp383

$\mathrm{Wu}$, T. D., and Watanabe, C. K. (2005). GMAP: a genomic mapping and alignment program for mRNA and EST sequences. Bioinformatics 21, 1859-1875.

Wu, W., Zong, J., Wei, N., Cheng, J., Zhou, X., Cheng, Y., et al. (2018). A constructing comprehensive splice site method for detecting alternative splicing events. Brief. Bioinform. 19, 905-917. doi: 10.1093/bib/bbx034

Zhang, J., Chiodini, R., Badr, A., and Zhang, G. (2011). The impact of next-generation sequencing on genomics. J. Genet. Genomics 38, 95-109. doi: $10.1016 /$ j.jgg.2011.02.003
Zhu, F. Y., Chen, M. X., Ye, N. H., Shi, L., Ma, K. L., Yang, J. F., et al. (2017). Proteogenomic analysis reveals alternative splicing and translation as part of the abscisic acid response in Arabidopsis seedlings. Plant J. 91, 518-533. doi: 10.1111/ tpj.13571

Zhu, S., Qing, T., Zheng, Y., Jin, L., and Shi, L. (2017). Advances in single-cell RNA sequencing and its applications in cancer research. Oncotarget 8, 53763-53779. doi: 10.18632/oncotarget.17893

Conflict of Interest Statement: The authors declare that the research was conducted in the absence of any commercial or financial relationships that could be construed as a potential conflict of interest.

Copyright () 2019 Wang, Kumar, Olson and Ware. This is an open-access article distributed under the terms of the Creative Commons Attribution License (CC BY). The use, distribution or reproduction in other forums is permitted, provided the original author(s) and the copyright owner(s) are credited and that the original publication in this journal is cited, in accordance with accepted academic practice. No use, distribution or reproduction is permitted which does not comply with these terms. 